\title{
Laboratory experiments simulating electron cyclotron masers in space
}

\author{
K. Ronald ${ }^{1}$, D.C. Speirs ${ }^{1}$, M. King ${ }^{1}$, T. Heelis ${ }^{1}$, S.L. McConville ${ }^{1}$, K.M. Gillespie ${ }^{1}$, R. Bingham ${ }^{1,2}$, \\ C.W. Robertson ${ }^{1}$, A.W. Cross ${ }^{1}$, A.D.R. Phelps ${ }^{1}$ \\ ${ }^{1}$ Department of Physics and SUPA, University of Strathclyde, Glasgow G4 0NG, Scotland, UK \\ a.d.r.phelps@strath.ac.uk \\ ${ }^{2}$ Rutherford Appleton Laboratory, Chilton, Didcot, Oxon, England, OX11 0QX, England, UK
}

The study of kinetic instabilities of the electron cyclotron family driven by anisotropic velocity distributions is important, as these instabilities occur in many situations and in particular there are many examples of their occurrence in space plasmas.

There is a well-established and very successful programme of research on this topic at IAP, Nizhny Novgorod [1]. Some of the series of publications from the IAP group are cited in a helpful recent publication by Shalashov et al. [2]. At the University of Strathclyde in Glasgow there has also been continuing interest in this subject for several years [3-11]. In this paper some of the results from laboratory experiments carried out at the University of Strathclyde are presented reproducing non-thermal cyclotron radio emission mechanisms associated with the Earth's auroral region, known as Auroral Kilometric Radiation (AKR). Similar mechanisms are thought to be responsible for some of the cyclotron emissions observed from more distant astrophysical environments.

These experiments were designed and carried out to benchmark PiC simulations of the non-linear, plasma mediated, beam wave interactions associated with the generation of Auroral Kilometric Radiation. A plasma column (20 cm to $1 \mathrm{~m}$ in length) was required inside a cylindrical waveguide having a diameter $\sim 5 \mathrm{~cm}$. This tightly constrained the dimensions allowed for the plasma source. To meet this constraint, two Penning-discharge configurations were designed. The electrons in a Penning discharge are insulated from the cylindrical anode (the waveguide) as it is linked by a magnetic field whilst the ends of the anode cylinder are closed by cathode electrodes, providing extended electron path lengths, allowing the discharge to be maintained at low pressures $\left(\sim 10^{-3} \mathrm{mB}\right)$. When a bias of a few $\mathrm{kV}$ was applied to the anode, the discharge ignited, establishing the plasma column. Up to a few 10 's $\mathrm{mA}$ were provided to the discharge in either a partially-modulating or continuous mode.

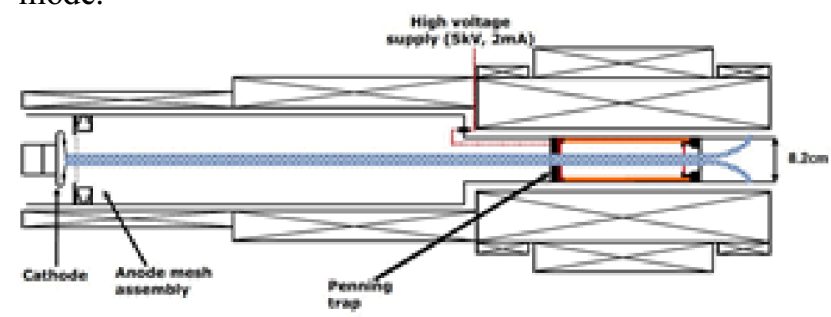

Fig. 1. Drawing of the low current trap and its location in the apparatus

The plasma density at the end of the column was inferred by observing the spectrum of plasma oscillations using a spectrum analyser connected to a small electric dipole antenna polarised along the bias magnetic field, thus avoiding problematic interpretation of the Langmuir probe IV trace caused by the strong magnetic field. The plasma density inferred at the end of the trap was found to vary with discharge current: $1 \times 10^{15} \mathrm{~m}^{-3}$ for $\sim 3 \mathrm{~mA}$ discharge current in a $20 \mathrm{~cm}$-long trap and $1 \times 10^{16} \mathrm{~m}^{-3}$ for a $20 \mathrm{~mA}$ current in a 1m-long trap.

To support this estimate of the plasma density at the end of the $1 \mathrm{~m}$ long plasma, and to determine the density averaged along the plasma column, an interferometric technique was implemented using a chirping microwave signal around $9.5 \mathrm{GHz}$ and simultaneously measuring the phase shifts in the $\mathrm{R}$ and $\mathrm{L}$ circularly polarised $\mathrm{TE}_{11}$ modes using a vector network analyser over a wide range of currents. At $20 \mathrm{~mA}$ the average density was estimated to be approaching $\sim 4 \times 10^{16} \mathrm{~m}^{-3}$.

Numerical PiC simulations carried out in previous research have helped to confirm the instability leading to the emissions is driven by a horseshoe electron velocity distribution.

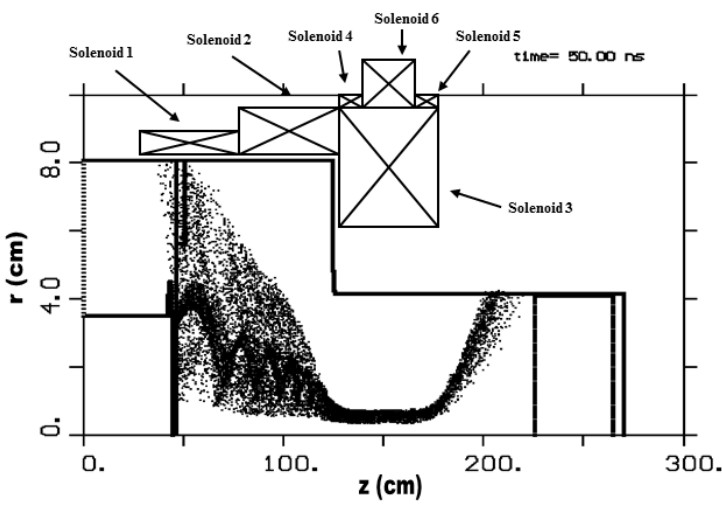

Fig. 2. The Configuration used in the KARAT PiC simulations. Note that the axial scale and the radial scale shown here are intentionally different

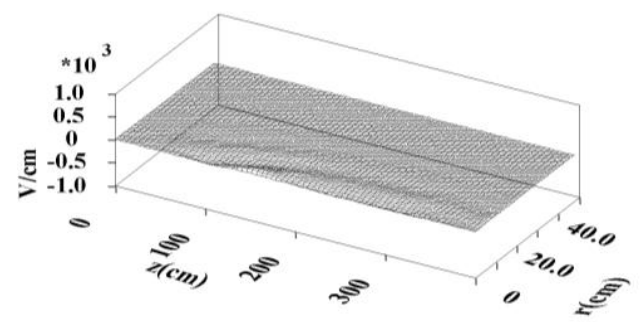

Fig. 3. 3D contour plot of $E_{\theta}$ within an unbounded simulation geometry at time $t=200 \mathrm{~ns}$. Backward wave propagation is evident

The PiC simulations that were undertaken using software, including KARAT, have reproduced the EM wave emission observed in the experiment using a bounded 
geometry as shown in Fig. 2 and have also been able to simulate the wave production in an unbound environment [10] representative of the magnetosphere, as shown in Fig. 3. Some of these experiments and simulations have revealed that the instability has a significant negative axial wavenumber (backward wave component) [10, 11]. The backward wave character of the emission is evidenced by an oblique wave front propagation angle with respect to the axis of the system. The magnitude of the negative axial wavenumber reduces with increasing energy spread.

\section{Acknowledgements}

The UK Engineering and Physical Research Council (EPSRC) is thanked for supporting this research. David Barclay provided valuable technical assistance.

\section{References}

1. Bespalov, P.A. and Trakhtengerts, V.Yu. in Reviews of Plasma Physics, edited by M. A. Leontovich. New York: Consultants Bureau. 1986. V.10; Trakhtengerts, V.Yu. and Rycroft, M.J. Whistler and Alfven Mode Cyclotron Masers in Space. New York: Cambridge University Press. 2008.

2. Shalashov, A.G., Viktorov, M.E., Mansfeld, D.A. and Golubev, S.V. Kinetic instabilities in a mirror-confined plasma sustained by high-power microwave radiation // Phys. Plasmas. 2017. V. 24, 032111. P. 1-13.

3. Speirs, D.C., Vorgul, I., Ronald, K., Bingham, R., Cairns, R.A., Phelps, A.D.R., Kellett, B.J., Cross, A.W., Whyte, C.G., and Robertson, C. A laboratory experiment to investigate auroral kilometric radiation emission mechanisms // J. Plasma Phys. 2005. V. 71. No.5 . P. 665-674.

4. Ronald, K., Speirs, D.C., McConville, S.L., Phelps, A.D.R., Robertson, C.W., Whyte, C.G., He, W., Gillespie, K.M., Cross, A.W., and Bingham, $R$. Radio frequency resonator struc- ture and diagnostic measurements for a laboratory simulation of Auroral Kilometric Radiation // Phys. Plasmas. 2008. V. 15. No. 5. 056503. P. 1-8.

5. Speirs, D.C., McConville, S.L., Gillespie, K.M., Ronald, K., Phelps, A.D.R., Cross, A.W., Bingham, R., Robertson, C.W., Whyte, C.G., Vorgul, I., Cairns, R.A., and Kellett, B.J. Numerical simulation of auroral cyclotron maser processes // Plasma Phys. Control. Fusion. 2008. V. 50. No. 7. 074011. P. 1-15.

6. Gillespie, K.M., Speirs, D.C., Ronald, K., McConville, S. L., Phelps, A.D.R., Bingham, R., Cross, A.W., Robertson, C.W., Whyte, C.G., He, W., Vorgul, I., Cairns, R.A., and Kellett, B.J. 3D PiC code simulations for a laboratory experimental investigation of Auroral Kilometric Radiation mechanisms // Plasma Phys. Control. Fusion. 2008. V. 50. No. 12. 124038. P. 1-11.

7. Ronald, K., Speirs, D.C., McConville, S.L., Gillespie, K.M., Phelps, A.D.R., Bingham, R., Vorgul, I., Cairns, R.A., Cross, A.W., Robertson, C.W., Whyte, C.G., He, W., and Kellett, B.J. Auroral magnetospheric cyclotron emission processes: numerical and experimental simulations // Plasma Phys. Control. Fusion. 2011. V. 53. No. 7. 074015. P. 1-11.

8. Vorgul, I., Kellett, B.J., Cairns, R.A., Bingham, R., Ronald, K., Speirs, D.C., McConville, S.L., Gillespie, K.M., and Phelps, A.D.R. Cyclotron maser emission: Stars, planets, and laboratory // Phys. Plasmas. 2011. V. 18, No. 5. 056501. P. 1-7.

9. Bingham, R., Speirs, D.C., Kellett, B.J., Vorgul, I., McConville, S.L., Cairns, R.A., Cross, A.W., Phelps, A.D.R., and Ronald, $K$. Laboratory astrophysics: Investigation of planetary and astrophysical maser emission // Space Science Reviews. 2013. V. 178. No. 2-4. P. 695-713.

10. Speirs, D.C., McConville, S.L., Gillespie, K.M., Phelps, A.D.R., and Ronald, K. Numerical simulations of unbounded cyclotron-maser emissions // J. Plasma Phys. 2013. V.79. No. 6. P. 999-1001.

11. Speirs, D.C., Bingham, R., Cairns, R.A., Vorgul, I., Kellett, B.J., Phelps, A.D.R., and Ronald, K. Backward Wave Cyclotron-Maser Emission in the Auroral Magnetosphere // Phys. Rev. Lett. 2014. V. 113. No. 15. 155002. P. 1-5. 